\title{
LA OPERACIÓN DEL DISPOSITIVO DE SEXUALIDAD EN LAS IDENTIDADES EMOCIONALES ATRIBUIDAS A LOS NIÑOS EN LOS LIBROS DE LECTURA ESPAÑOLES Y COLOMBIANOS EN LA PRIMERA MITAD DEL SIGLO XX ${ }^{1}$
}

\author{
Federico Guillermo Serrano-López ${ }^{2}$
}

\section{RESUMEN}

En los libros de lectura para la escuela primaria españoles y colombianos de la primera mitad del siglo XX es frecuente atribuir a los protagonistas de las lecturas una identidad emocional fija y un fondo instintivo que son revelados por sus comportamientos. Se estudiará la relación del

1 El presente trabajo hace parte del proyecto $X X X$ que se ha puesto como objetivo general "Analizar históricamente desde la relación pedagogía-ciencias-saberes, el proceso de configuración del campo de las disciplinas y saberes escolares en Colombia, con el objeto de hacer visible las formas de apropiación de las ciencias y los saberes en la enseñanza en Colombia." Particularmente, contribuye al objetivo específico de "Examinar los contenidos de la preparación para la sexualidad seleccionados por la escuela colombiana para hacer visible la configuración de un saber escolar sobre el género, las emociones, los deseos y placeres."

2 Universidad del Norte (Uninorte), Barranquilla, Colombia. 
uso de estas atribuciones con las estrategias para preparar la subjetividad infantil en cuanto a sus reacciones frente al deseo, el placer y las relaciones sociales. Se sostiene que esas estrategias hacen parte de la operación del dispositivo de sexualidad (conforme lo entiende Foucault). Se concluye que el sexo parece jugar allí el rol de determinación fundamental del instinto y el yo, de allí la relevancia que cobra la preparación para la sexualidad en la estructuración del proyecto escolar.

Palabras clave: historia de la sexualidad, España, Colombia, identidad, emociones, manuales escolares.

\title{
A OPERAÇÃO DO DISPOSITIVO DE SEXUALIDADE NAS IDENTIDADES EMOCIONAIS ATRIBUÍDAS A CRIANÇAS EM LIVROS DE LEITURA ESPANHÓIS E COLOMBIANOS NA PRIMEIRA METADE DO SÉCULO XX
}

\section{RESUMO}

Nos livros de leitura espanhois e colombianos da escola primária da primeira metade do século 20 é comum atribuir aos seus protagonistas uma identidade emocional fixa e um fundo instintivo que são revelados pelos seus comportamentos. Será estudada a relação do uso dessas atribuições com as estratégias para preparar a subjetividade infantil em relação às suas reações ao desejo, ao prazer e às relações sociais. Argumenta-se que essas estratégias fazem parte da operação do dispositivo de sexualidade (como é entendido por Foucault). Conclui-se que o sexo parece desempenhar o papel de determinação fundamental do instinto e do eu, daí a relevância da preparação para a sexualidade na estruturação do projeto escolar.

Palavras-chave: história da sexualidade, Espanha, Colômbia, identidade, emoções, manuais escolares.

\section{THE OPERATION OF THE DEVICE OF SEXUALITY IN THE EMOTIONAL IDENTITIES ATTRIBUTED TO CHILDREN, IN SPANISH AND COLOMBIAN PRIMARY SCHOOL'S READING BOOKS, IN THE FIRST HALF OF THE 20TH CENTURY}

\begin{abstract}
In Spanish and Colombian primary school's reading books of the first half of the 2oth century, it is common to attribute a fixed emotional identity and an instinctive background, revealed by their behaviors, to the text's protagonists. This paper studies the relationship between the usage of these attributions and the strategies that prepare the infantile subjectivity in terms of their reactions to desire, pleasure and social relationships. It is argued that these strategies are part of the operation of the device of sexuality (as Foucault understands it). The conclusion states that sex seems to play the role of fundamental determination of the instinct and the self, hence the relevance of sexuality preparation in structuring the school project.
\end{abstract}

Keywords: history of sexuality, Spain, Colombia, identity, emotions, textbooks. 


\section{L'OPÉRATION DU DISPOSITIF DE SEXUALITÉ DANS \\ LES IDENTITÉS ÉMOTIONNELLES ATTRIBUÉES AUX \\ ENFANTS DANS LES LIVRES DE LECTURE ESPAGNOLS \\ ET COLOMBIENS DANS LA PREMIÈRE MOITIÉ DU XXE SIÈCLE}

\section{RÉSUMÉ}

Dans les livres de lecture espagnols et colombiens pour l'école primaire de la première moitié du XXe siècle, il est fréquent d'attribuer aux protagonistes des lectures une identité émotionnelle fixe et un fond instinctif qui sont révélés par leurs comportements. On étudiera la relation de l'usage de ces attributions avec les stratégies pour préparer la subjectivité infantile en ce qui concerne ses réactions face au désir, au plaisir et aux relations sociales. On soutient que ces stratégies font partie de l'opération du dispositif de sexualité (selon Foucault). On conclut qu'ici le sexe semble jouer le rôle de détermination fondamentale de l'instinct et du moi, c'est qui met en évidence l'importance de la préparation pour la sexualité dans la structuration du projet scolaire.

Mots-clés: histoire de la sexualité, Espagne, Colombie, identité, émotions, manuels scolaires. 


\section{INTRODUCCIÓN}

La tesis de este artículo es que la preparación para la sexualidad jugó un papel decisivo en la estructuración del proyecto escolar de la primera mitad del siglo XX en España y Colombia, a pesar de que la educación sexual nunca tuvo, en el periodo, la condición de disciplina escolar y de que, como saber, su presencia en la escuela fue latente y difusa3. Esta relevancia de la preparación para la sexualidad en la escuela fue el resultado de la operación del dispositivo de sexualidad que tuvo diversas manifestaciones y cuya presencia fue transversal a toda la enseñanza, en la escuela y fuera de ella4. Se entiende por dispositivo de sexualidad, siguiendo a Michel Foucault (1976, p. 129-130), el conjunto de estrategias ejecutadas por los agentes de poder que usaron al sexo como pretexto y soporte para intensificar la vigilancia de los individuos por parte de todo el aparato social y lograr la participación de los sujetos en investigarse a sí mismos y controlar su conducta.

Las evidencias de las afirmaciones anteriores han sido investigadas, en los trabajos mencionados en la última nota, por medio de la revisión de los discursos sobre el cuerpo y la sexualidad en el periodo y el examen de fuentes en las que se hagan manifiestos los valores considerados dignos de ser enseñados, las actitudes frente a los niños, algunos ejemplos concretos utilizados para ilustrar la enseñanza, el lenguaje y la atmósfera misma del espacio escolar. En todos estos sentidos, los manuales escolares son una fuente privilegiada como ventana para observar no solo la escuela y sus satélites naturales: el espacio familiar, los maestros y las actitudes frente a lo que representaba lo diferente del medio infantil, principalmente la calle, sus personajes y peligros. Entre los manuales escolares se han seleccionado como objeto de estudio los libros de

\footnotetext{
3 En ambos países se hallan estudios que abordan temas relacionados con la educación sexual en la primera mitad del siglo XX. En España: Guereña, 2011; Vásquez y Moreno, 1996; 1997; Seoane, 2006; Pérez, 1993; 1996. En Colombia: Bacca y Ramírez, 2003; Pedraza, 1999; Urrego, 1997; Sáenz, Saldarriaga y Ospina, 1997; Noguera, 2003; Bidegaín, 1995; Roa, 2017.

4 Tal es la tesis de la investigación de la cual este artículo es un apartado. Ver: Serrano-López, 2013; Serrano-López, 2015a; Serrano-López, 2015b; Serrano-Lopez y Somoza-Rodríguez, 2017.
} 
lectura de la escuela primaria5; esto es, los textos para practicar la lectura comprensiva, principalmente en voz alta. Eran recopilaciones de textos de diferentes fuentes, géneros y estilos que, además de servir de ilustración del uso del idioma, ejemplificaban los valores y dejaban ver las reacciones sociales frente a las actuaciones infantiles. Tanto las autoridades escolares como los maestros y los autores de estos textos manifiestan que una de sus principales finalidades y rasgos de calidad consiste en afectar la emotividad infantil para lograr un aprendizaje profundo y duradero. Por tanto, los libros de lectura representan una fuente para conocer la estructura de valores de la época que los produjo, aunque es importante señalar dos limitaciones: primera, se encuentran en ellos principalmente los puntos de vista 'oficiales', que eran los que se solían seleccionar para agradar a las autoridades educativas, a los maestros y a los mismos padres de familia; segunda, no nos dicen cómo fueron leídos, ni sus efectos en los lectores. Sin embargo, su valor como documento histórico reside en que compendian qué se quiso enseñar para el sostenimiento del statu quo y, con esa salvedad, son una fuente útil para reconstruir parte del complejo entramado cultural de la escuela. Para este estudio fueron seleccionados 90 libros de lectura para la escuela primaria (67 españoles y 23 colombianos) usados en el periodo. La selección se hizo basándose en la importancia de las casas editoriales que los editaron, el número de ediciones del mismo manual, la importancia relativa de los autores y los juicios emitidos al respecto por autoridades escolares y por los mismos maestros. Se compararon los libros de lectura españoles con los colombianos, pues hubo en la época un intenso intercambio entre España e Iberoamérica en relación con los manuales escolares, de modo que las intenciones pedagógicas, los tipos de lecturas, el lenguaje y las estrategias didácticas son semejantes y elocuentes de preocupaciones comunes. Sin embargo, las diferencias entre los textos de los dos países enriquecieron el análisis al poner en alto distintas intenciones,

\footnotetext{
5 Respecto de la historia de los manuales escolares en España: Escolano, 1997; Tiana-Ferrer, 1999; 2000; Hernández Díaz, 1994; Villalaín, 1997. Sobre libros escolares en Colombia: Guereña, Ossenbach y Del Pozo, 2005; Ossenbach y Somoza-Rodríguez, 2001; CondeCalderón, 2001; Rincón-Verdugo, 2003; Pinilla, Sauza y Herrera, 2003.
} 
estrategias y énfasis de los autores y editores que permiten identificar diferencias en relación con la comprensión del cuerpo, la sexualidad y los fines del proyecto educativo.

El reto de la investigación estaba en encontrar el funcionamiento del dispositivo de sexualidad en los libros de lectura de periodo, en los que muy poco o nada se habla de sexo (aunque se lo menciona equívocamente mucho más de lo que generalmente se cree). Respecto de este tema se debe tener en cuenta que el dispositivo de sexualidad no se ve solo en los discursos sobre sexo, sino en las estrategias que se usaron para controlar a los individuos que tuvieron como pretexto el control de los placeres y deseos sexuales, así como en la valoración que se hacía de las personas a propósito de sus capacidades para gobernar sus instintos. En virtud de esto, el control de la sexualidad puede jugar un papel decisivo en la configuración de la subjetividad individual y social, aunque no se hable explícitamente de sexo. En atención a lo anterior, se sostiene que en los libros de lectura para los niños de la escuela primaria se muestra al dispositivo de sexualidad funcionando de forma muy intensa, aunque latente, en la estructuración de la subjetividad infantil a través de la vigilancia de las formas de placer y deseo y en las actitudes aceptadas o censuradas para el disfrute.

El presente artículo se concentrará en hacer visible un mecanismo clave dentro de las estrategias utilizadas en los libros de lectura para controlar las actitudes frente a los deseos y placeres. Se trata de la tendencia, predominante en los libros españoles y colombianos de las primeras tres décadas del siglo XX, a identificar en los sujetos un fondo sustancial fijo en virtud del cual se pretendía explicar las tendencias comportamentales de los niños; por ejemplo, cuando a partir de una acción generosa un niño mostraba tener 'buen corazón' o cuando por ser cruel con los animales revelaba que tenía 'malos sentimientos'. En este artículo se hace, pues, seguimiento al tratamiento de las historias, poemas y dibujos en los libros de lectura en las que sus autores señalan unos comportamientos como manifestaciones profundas de una identidad emocional 
$\mathrm{y}$ un fondo instintivo que constituyen la subjetividad de los individuos. Al examinar dichos casos se hará patente que el sexo juega un papel protagónico en cómo se concibió la subjetividad profunda de los niños, pues el deseo sexual parece jugar el rol de determinación primera de las acciones irracionales humanas, y no solo a nivel individual, sino familiar y social. Y así, en todos los temas donde se señalan estas manifestaciones profundas de la subjetividad (el buen o el mal corazón, los buenos y malos sentimientos, las pasiones y los instintos) se manifiesta una forma de comprender el fenómeno del sexo, y de su relevancia como clave de interpretación sobre cómo fueron concebidos los sujetos y la sociedad.

\section{EL ALMA Y LA ÍNDOLE INDIVIDUAL}

En gran parte de los textos del periodo se asume que la esencia de la subjetividad reside en el alma. Cada alma posee una índole propia, una impronta individual que la hace tender hacia el bien o el mal. En concordancia con la tradición cristiana, el alma es diferente del cuerpo y, sin embargo, son los comportamientos concretos del cuerpo, así como la apariencia física, los que manifiestan la índole particular de esa alma y las tendencias de las facultades que la componen: los sentimientos, la imaginación, la voluntad y la inteligencia. "Nuestro exterior manifiesta las disposiciones de nuestra alma y mueve ora a estima, ora a desprecio, según que aquél sea correcto o no." (VALENTÍn O EL NIÑO BIEN EDUCADO, 19??, p. 17). Así, los personajes de las lecturas expresan, con sus palabras y acciones, buenos o malos sentimientos, una imaginación desbocada, una voluntad firme o una inteligencia aguda, etcétera. 


\section{EL BUEN CORAZÓN Y LOS BUENOS SENTIMIENTOS}

En los textos se utilizaron numerosas expresiones que señalan los signos de la índole del sujeto: el buen o mal corazón, los buenos y malos sentimientos, las buenas y malas inclinaciones. Con estas expresiones se sintetiza la sensibilidad moral del sujeto, sus tendencias afectivas y estéticas, y la disposición de su voluntad frente a la moralidad. Se trata de conceptos complejos cuyo sentido se va revelando al lector en los contextos en los que se aplican, pero en todos ellos es patente que ejercen la función de definir a la persona desde el punto de vista moral y afectivo. La calificación que estos conceptos señalan es fundamental y definitiva sobre el ser individual, y una vez la comunidad, encarnada en la voz del maestro o de los mismos padres, ha juzgado sobre esa índole, su veredicto parece ser inapelable e imborrable.

Los temas del buen corazón y los buenos sentimientos son más frecuentes en los manuales de las primeras dos décadas del siglo XX en España, pero su mención se conserva, aunque de manera menos habitual, hasta los años cincuenta. En los textos colombianos está presente también esta terminología de forma esporádica a lo largo de todo el periodo; pero allí es más frecuente que se hable de buenos o malos instintos.

La marca más frecuente del buen corazón y los buenos sentimientos estaba en ser afectuoso con los animales y los gestos de compasión con los débiles, los pobres y los "desgraciados", que era la forma de llamar a las personas con limitaciones físicas. Así mismo, eran tomados como señales de un buen corazón el desinterés por el dinero y las recompensas frente al cumplimiento del deber; la generosidad, solidaridad y disposición al sacrificio desinteresado con los familiares y amigos; la preocupación por el bienestar ajeno; el respeto por los ancianos y los niños; el amor a los padres; la obediencia y sumisión ante los superiores (padres, maestros, sacerdotes, jefes y autoridades civiles); la disposición a perdonar. Por último, son signos claves de buenos sentimientos la piedad sincera, el candor, la ternura, la inocencia y, en general, 
la bondad.

El mal corazón se reconoce por las conductas inversas a las enumeradas antes. La más frecuente es el maltrato a los animales, pues en ella hay dos agravantes: uno, se hace un mal fortuito, y dos, la víctima es inocente. Este primer elemento, hacer un mal innecesario, hace pensar que se encuentra placer en el mal mismo y en el dolor ajeno. Por las mismas razones, burlarse de los que sufren es signo de malas inclinaciones. Un segundo grupo de factores delatores del mal corazón son los relacionados con la desobediencia, el disimulo de las faltas y la rebeldía con los agentes de autoridad. En tercer lugar, todas las formas de egoísmo y de excesiva preocupación por los propios beneficios: la avaricia, la poca caridad, la falta de solidaridad con amigos y familiares, la tendencia a encapricharse con cosas materiales y la vanidad. En cuarto lugar, muestran malos sentimientos las personas que hacen gestos que delatan una preocupación perversa por las acciones y beneficios de otras personas: la venganza, los celos, la envidia y la ira. Por último, las marcas relacionadas con los placeres y deseos sexuales son reveladoras de malos sentimientos y señalan un alma impura: miradas insinuantes, conversaciones impuras, chistes de doble sentido y risas licenciosas.

Buen y mal corazón, inclinaciones y sentimientos pueden transparentarse en el físico. Así lo afirman en el texto colombiano Mi cuarto libro de lectura: "Nuestra fisonomía nos delata; los gestos, las sonrisas, las miradas, hablan más claramente que los labios. Llevamos nuestra historia escrita sobre la frente, y en ella lee quien quiere hacerlo" (MARÍN; CANO, 1952, p. 193). El buen corazón se manifiesta habitualmente en una belleza angelical. Se lee en la lectura "El rostro" del texto español Valentín o el niño bien educado: "iQue hermoso es el rostro de un niño o de un joven verdaderamente virtuoso! ¡Es el encanto de los hombres y el embeleso de los ángeles!" (VALENTÍN O EL NIÑO BIEN EDUCADO, 19??, p. 44). Y hacen inmediatamente la descripción de la compostura de San Juan Berchmans: 
Era tal la serenidad de su frente, la limpidez de su mirada, la habitual sonrisa de sus labios, que todo revelaba en él un alma inocente y pura, justificando así la opinión en que le tenían sus compañeros, quienes a boca llena le apellidaban el ángel. Según testimonio de los que vivieron con él, nunca le vieron perder la calma, ni demostrar la más ligera contrariedad; antes, por el contrario, sonreía con especial amabilidad cuando se le ofrecía alguna ocasión de impaciencia. La expresión de su rostro era la misma en el recreo que en el estudio, en el jardín que en la iglesia.

La modestia de sus ojos era tan singular que siempre los mantenía bajos, hasta el punto de costarle trabajo levantarlos del suelo, y sus compañeros nunca pudieron saber de qué color eran.

No movía ninguno de sus miembros sin necesidad. Jamás causó disgusto a nadie, y tanto se vigilaba en esto, que no le vieron hacer nada que pudiera molestar en lo más mínimo a los demás.

De tal modo vigilaba sus palabras, que se hubiera dicho que no sabía hablar más que de cosas buenas y piadosas.

Tomemos, pues, por modelo a este admirable joven e imitemos, sobre todo, su recato y angelical pureza (VALENTÍN O EL NIÑO BIEN EDUCADO, 19??, p. 44-45).

En ocasiones la belleza del niño bueno e inocente coincide con que tiene piel blanca, ojos azules y cabello rubio ensortijado, uniendo así el prejuicio racial con la representación tradicional de los ángeles. Sin embargo, se hallan también niños de buen corazón de cabello negro (y piel blanca), y se encuentran ejemplos de niños "desgraciados" (con limitaciones físicas), mostrados expresando buenos sentimientos.

Por su parte, el mal corazón tiene también manifestaciones físicas. Así, se encuentran casos de personajes descritos como físicamente feos que cometen actos que manifiestan su mal corazón. Por ejemplo, en la lectura "El dedal de marfil" del libro español Mi costurero, una niña bizca, que tiene "torcidos los ojos y el alma”, intenta apoderarse del dedal de Panchita, una niña de buen corazón. Cuando ésta se niega:

"La bizca" se puso roja de cólera. Precisamente aquel dedal era su obsesión y la envidia subía en su pecho aplastándolo. A "la bizca" la relumbraron los ojos con luces diabólicas... se agacho y... de un tirón 
Sin embargo, sobre la niña bonita, pero vanidosa, cae también una sospecha, especialmente en los manuales del inicio del periodo. Vemos ejemplo de ello el manual español Mi primer manuscrito, en el que describen a una niña de malas inclinaciones enfatizando su frivolidad: "Los pobres le repugnaban, y solo pensaba en la satisfacción de sus locuras y vanidades. Cifraba todos sus goces en el estreno de un traje, en la compra de un sombrero o en asistir a una función de teatro". A lo que su mamá le dice: "Los trajes vistosos, los paseos callejeros, las joyas, los teatros solo pregonan nuestra vanidad y la ausencia de los sentimientos que nos acercan a Dios" (DALMAU, 1925, p. 103-105).

Las prácticas sexuales ilícitas, particularmente la masturbación, también dejan marcas fisiológicas que afean: "Nada desagrada tanto como el aspecto del joven vicioso porque por más que quiera evitarlo, su cara aparece marcada con el sello de la deshonestidad y de la desvergüenza” (VALENTíN O EL NIÑO BIEN EDUCADO, 19??, p. 42). Esa fealdad no siempre se muestra de modo constante, sino que puede aparecer súbitamente reflejada en el rostro, como sucede en las miradas licenciosas. Los inesperados resplandores en los que se manifiesta algún aspecto del sexo se muestran como ocasiones para que emerja un ser bestializado cuyo rostro y actitud corporal son detestables, pero sobre los cuales hay una evidente atracción. Ese fondo bestial es, sin embargo, paradójico, porque si bien delata a los malvados, se teme que todos de algún modo sean semejantes y, por tanto, la educación debe cumplir una labor domesticadora.

\section{LAS PASIONES}

La subjetividad individual puede verse afectada por unos deseos y sentimientos de especial intensidad que no son pasajeros, sino que, de forma 
persistente, aunque con intensidad variable en diferentes momentos de la vida, se imponen y determinan el juicio de un individuo opacando su inteligencia y su voluntad; esas son las pasiones. Así son definidas en el libro español Escenas de familia:

\begin{abstract}
Las pasiones, que son el amor o la antipatía a determinadas personas y objetos, la envidia, la cólera, etcétera, contribuyen poderosamente a viciar o extraviar nuestro juicio: por ejemplo, una niña tiene una amiguita muy querida, y aunque los demás reconozcan en la última que es perezosa o desaplicada, ella la juzgará perfecta (PASCUAL DE SAN JUAN, 1891, p. 275).
\end{abstract}

En las pasiones las motivaciones son profundas y están vinculadas con la historia del sujeto y con las tendencias de su índole. Por tanto, son diferentes de los caprichos, que son deseos intensos, pero ocasionales y de poca importancia (por ejemplo, comprar una muñeca). En las pasiones se involucra el núcleo del sujeto y sus efectos son de mayor trascendencia (por ejemplo, robar por ambición).

En ocasiones las pasiones son valoradas positivamente, como cuando motivan la composición de una obra de arte; pero en la mayoría de los casos son vistas como causas de aberraciones y violencia. El siguiente fragmento se hallan en el texto español El amigo: "[Al palacio de justicia van a dar los] soberbios, avaros, envidiosos, iracundos, golosos, disolutos y holgazanes, que se dejan dominar por las malas pasiones, arrastrar por los vicios y guiar por las malas compañías" (PAZZI, 1921, p. 232). El peligro que encarnan estriba en su rápida capacidad de crecimiento y fortalecimiento que hace muy difícil arrancarlas si se les ha dejado crecer, así sea un poco; de allí su cercanía con los vicios. Así mismo, los malos sentimientos, a los que todas las personas son susceptibles en algún momento, si se convierten en hábitos, se fortalecen hasta formar malas pasiones.

La misión de la educación consiste, por tanto, en entrenar al niño para 
controlar los malos sentimientos antes de que se conviertan en pasiones. En el texto Alma española, para ilustrar el efecto de le educación narran una anécdota, atribuida a Licurgo, en la que él puso frente a frente a dos perros y dos liebres. Soltó el primer perro que despedazó a la primera liebre. Luego soltó el segundo perro, quien jugó con la liebre:

\footnotetext{
Este perro y dicha liebre se habían criado juntos bajo la inspección de Licurgo, mientras que el otro, no acostumbrado a la compañía de las liebres, esto es, no educado, solo obedecía a sus brutales instintos, como el hombre cuyas pasiones no enfrena la educación (ARIAS, 1939, p. 246).
}

Se evidencia, por tanto, la creencia en un estado salvaje anterior a las influencias de la cultura. Como lo humano implica la vida en comunidad, y consecuentemente, la domesticación de las pasiones, en el estado salvaje el hombre es una bestia, lo cual se aplica también a los pueblos alejados de la educación civilizadora. A la inversa, un hombre educado que manifiesta estar dominado por una pasión tiene la determinación y la fuerza de un animal, lo cual lo puede llevar a realizar hazañas grandiosas. El caso ejemplar de expresión animal de una pasión es la ira. Sin embargo, hay, además del que abiertamente muestra sus pasiones, el que las disimula y que es por eso, peligroso y culpable. En ese caso se puede hablar de pasiones ruines en las que se une, a la profunda determinación animal, el cálculo para obtener la satisfacción de fines oscuros. Tal es el caso de los envidiosos, los hipócritas y los calumniadores. También se califican como ruines las pasiones cuyo móvil es un exceso en el deseo: la gula, la ambición y la lujuria.

En tanto que afectan a los individuos las pasiones pueden ser perjudiciales; sin embargo, son mucho más temidas cuando dominan a los pueblos. Y así como el dominio de las pasiones es signo distintivo de virtudes en los individuos, en los pueblos ello constituye el éxito del proyecto de civilización. En tal sentido es muy elocuente la cita que hacen de un fragmento de un texto 
de José Zorrilla en el libro de lectura español Deberes:

\begin{abstract}
Quiero un pueblo de actitudes capaces de iniciativas cristianas, serias y activas y de cívicas virtudes. Pueblo, en fin, con las ventajas de las practicas modernas; con más granjas que tabernas, con más virtudes que alhajas; sin viles pasiones bajas, sin resabios ni secuelas, con más libros que barajas, más aperos que vihuelas; con muchísimas escuelas y poquísimas navajas (DALMAU, 1906, p. 159).
\end{abstract}

Las pasiones ruines propician un egoísmo que disuelve los lazos de la solidaridad y la comunidad. Sin embargo, la fuerza del carácter puede transformar los deseos egoístas en pasiones nobles y virtudes heroicas de las que dan muestras individuos y pueblos enteros; sobre todo, en situaciones extremas, como la guerra, en la que los espíritus más fuertes muestran la firmeza de su talante, y la integridad moral de la voluntad se puede convertir en una determinación tan sólida y poderosa que transforme cualquier pasión en una virtud. A pesar de esta posibilidad, la mayoría de las menciones a las pasiones son para condenarlas como causa de desgracias. Hacia el final del periodo, en ambos países, la palabra deja de ser frecuente en los libros no religiosos, pero se mantiene como motivo de censura en los religiosos.

\title{
LOS INSTINTOS
}

Con el desarrollo del naturalismo en el siglo XIX y los descubrimientos sobre la herencia, cobró relevancia el tema del instinto como tendencia espontánea del comportamiento heredada por las características biológicas de los padres. Al aplicar ese tema a la moralidad del individuo en los libros de lectura, mayoritariamente en aquellos escritos a finales del siglo XIX y comienzos del siglo XX, en ambos países, se encuentra a los buenos o malos instintos (en algunos hablan de impulsos); o sea, resortes para que el individuo espontáneamente haga el bien o el mal, y que manifiestan una tendencia innata 
heredada, que se acentúa, o se atenúa, con la educación. Dichos instintos no solo son marcas individuales, sino que se manifiestan en las familias y los pueblos; y a los mismos se les achaca ser la causa del estado del desarrollo material y moral de países y razas.

$\mathrm{Al}$ igual que las pasiones, los instintos tienen valoraciones positivas y negativas; pero, a diferencia de ellas, es mucho mayor el desarrollo del aspecto positivo de los instintos. $Y$ entre los instintos apreciados, el mayor de todos es el maternal que se manifiesta en los personajes femeninos más destacados: las madres, y entre ellas, la Virgen María. Adicionalmente, se hallan varias lecturas dedicadas a mostrar como las hembras de distintas especies demuestran un intenso deseo de ser madres y un espíritu de sacrificio desinteresado por sus crías. Del mismo modo, mujeres jóvenes y ancianas expresan, en numerosas lecturas, su disposición a la protección y el cuidado.

Entre tanto, en los hombres la manifestación fundamental del instinto se da en la virilidad, en una imagen de la masculinidad que se considera innata y surgida de las energías de la herencia racial, y que es afín a las misiones que, como género, cumplen los varones en la familia patriarcal. A este respecto se afirma lo siguiente en un libro de lectura colombiano:

Para el hombre, el ruido y las espinas de la gloria; para la mujer, las rosas y el sosiego del hogar; para él, el humo de la pólvora; para ella, el humero de la alhucema. Él destroza, ella conserva; el aja, ella limpia; él maldice, ella bendice; él reniega, ella ora (BERNAL, 1891, p. 187).

La disposición viril expresa la fortaleza del instinto sexual en los hombres y ello, en los manuales más cercanos al siglo XIX, permite explicar las indiscreciones y los comportamientos eventualmente ofensivos de los varones: 
En la medida en que la energía viril no es propia de las mujeres (de hecho, cuando una mujer muestra determinación se la compara con los hombres), no tienen pretexto para ser gobernadas por el apetito y, por tanto, no se les disculpa un tropiezo moral.

El instinto viril tiene su manifestación en la energía física, la cual, a su vez, tiene expresión en una disposición asertiva para la acción, que con frecuencia toma la forma de agresividad y violencia sobre personas y pueblos débiles. Esta virilidad arrogante fue una forma de comprender la masculinidad muy frecuente en los libros de lectura del primer franquismo.

En el mismo grupo de los instintos valorados positivamente, la disposición a la generosidad y a la captación del bien y de la belleza se consideraba que tenían un aspecto innato que, si bien se refinaba con la educación, provenía de unas tendencias instintivas de la naturaleza. Así, por ejemplo, en Escenas de familia explican el surgimiento de las ciencias basándose en los instintos generosos de la humanidad (PASCUAL DE SAN JUAN, 1891, p. 200). Y, al igual que en el caso del instinto maternal, estos instintos generosos no solo están en los seres humanos, sino que se ven también en los animales. También a los niños se les achaca el manifestar estas disposiciones. Así, por ejemplo, en el libro tercero de las Lecturas graduadas de F.T.D., texto publicado en ambos países, en una premiación escolar un niño le manda un beso a su madre con el diploma, y para el autor de la lectura ello revela "ese instinto de ángel que hace comprender al niño lo que es santo y bello" (LECTURAS GRADUADAS. LIBRO TERCERO, 1931, p. 71).

La contraparte, los malos instintos, no son necesariamente malos por sí mismos, pues cumplen una función natural, sino que son malos en exceso, en ocasiones inoportunas o con objetos inadecuados. El objeto de la educación no consiste en negar esos instintos, sino en regularlos para adaptarse a la vida 
social. Así, por ejemplo, dice en el Nuevo lector colombiano:

\begin{abstract}
Cuando se logra observar cotidianamente las normas protocolarias, se consigue el hábito benéfico de controlarse, de dominar los impulsos desbocados del instinto, lo que deja un producto de robustecimiento moral y de capacidad enérgica que acreditan a quien los conquista como elemento esencial del núcleo al que pertenece (CORTÁZAR, RENGIFO Y HERRERA, 1913, p. 201).
\end{abstract}

Sin embargo, en el descontrol instintivo se puede encontrar cierta gradación en la gravedad del tipo de exceso: entre los menos graves se ven manifestaciones instintivas socialmente censurables, como el egoísmo y la voracidad; pero el descontrol instintivo puede llegar hasta la manifestación de instintos de animales salvajes: los instintos violentos y, finalmente, los más graves de todos, los instintos asesinos, que pueden bestializar a una turba enardecida o a un hombre furioso.

El instinto sexual está ubicado entre los impulsos peligrosos propios de los animales. Así, por un lado, su descontrol representa un riesgo, y además se lo ve como un impulso para propiciar los excesos: la voracidad, el egoísmo, la violencia y el crimen. Sin embargo, también se supone al instinto sexual en la base de los instintos más valorados: la maternidad en la mujer y la virilidad en los varones. Este doble signo contradictorio se mantiene en los libros de lectura hasta los primeros años de la década de los cuarenta. De ahí en adelante, al igual que como sucedió con las pasiones, las menciones al instinto se hacen cada vez más breves e infrecuentes.

\title{
DETERMINISMO Y EDUCACIÓN FRENTE A LA ÍNDOLE Y LA HERENCIA
}

En los textos el tratamiento que reciben las determinaciones profundas del sujeto es problemático respecto a las posibilidades que tiene la educación 
para corregir las tendencias innatas. En numerosos manuales de finales del XIX y comienzos del XX se trata a los sujetos como sustancias fijas que no cambian en su esencia y que, por ello, están abocados a manifestar una naturaleza que los conduce fatalmente al bien o al mal, y consecuentemente, al éxito o al fracaso. Caso de ello se encuentra en el tercer libro de la serie española Pepe, de Raimundo Gómez Tutor. En él se presenta el ejemplo de Luis, un niño bueno que servía de comparación a los padres de otros niños para regañarlos cuando se portaban mal. Como era honrado, al crecer obtiene el premio de la riqueza y el aprecio de todos. Con su dinero brinda a sus padres muchas comodidades y luego muere y va al cielo. En contraste, en la página siguiente, aparece Crispín: "[...] reflejo del mal, o como si dijéramos del demonio" (GÓMEZ TUTOR, 190?, p. 40). Crispín es desobediente, se burla de los ancianos, de los sacerdotes, de las autoridades, de los maestros y de sus padres. Bebía desde que estaba en la escuela. Se hace soldado y lo meten al calabozo, luego a la cárcel por golpear a un superior. Al regresar al pueblo todos huyen de él. Asesina a una persona y lo condenan a muerte, pero Crispín no se arrepiente. Al final de la lectura preguntan: “¿Estará el alma de Crispín con la de Luis?” (GÓMEZ TUTOR, 190?, p. 40).

Como se ve, el aspecto decisivo que establece el límite entre la naturaleza buena y la mala es la actitud frente a la autoridad: el bueno obedece, el malo es rebelde. Así, al parecer, la educación solo tiene alcances entre aquellos predispuestos a la sumisión y a dominar sus deseos; los otros, los desobedientes e intemperantes, están malditos como Caín (relato que se cita en varios de los textos de este sub-periodo), y con ellos la educación es impotente.

Es importante resaltar que la posibilidad de adquirir el carácter sumiso depende de subordinar el placer al deber. Ello se ve en el siguiente texto colombiano:

Todos experimentamos que en nosotros hay dos hombres: uno inteligente, activo, de pensamientos elevados de deseos nobles, conforme a la razón, de proyectos arduos y grandiosos; otro, torpe, 
soñoliento de miras mezquinas, que se arrastra por el polvo cual inmundo reptil. [...] Para el segundo no hay el recuerdo de ayer ni la previsión de mañana; no hay más que lo presente, el goce de ahora; lo demás no existe. [...] Para todo adelanto sólido y estable conviene desarrollar al hombre noble, y sujetar y dirigir al innoble con la firmeza de la voluntad (BERNAL, 1891, p. 167).

Y entre los ámbitos que son definidos por el goce, el sexo es decisivo, pues es el que mayormente obstaculiza el dominio de los apetitos y la prueba desde la cual el sujeto logra, o no, hacerse un hombre. Pues el que prefirió el placer al deber, se comporta como un animal (un inmundo reptil). Así, el descontrol sexual va implícitamente asociado, en los varones, al salvajismo y al crimen; y en las mujeres, a la enfermedad y la prostitución; y por eso, allí donde aparece el sexo ilícito se puede reconocer claramente la maldad. Esto sucede en la lectura “Un niño malo” del Libro II de La escuela colombiana:

Juan es un muchacho que de todo se burla. No reza al acostarse, ni al
levantarse, ni oye misa con devoción, ni comulga sino cuando lo
obligan. Por eso el corazón de Juan está lleno de vicios. Allí se
extiende como mancha de tinta la impureza, que sube hasta la lengua
con palabras indecentes (RESTREPO MEJÍA, 1918, p. 43).

Ahora bien, dominarse a sí mismo es una disciplina de toda la vida; perderse, en cambio, es muy fácil, basta con una sola mancha, y en numerosos casos, como el ya citado de Crispín, cuando la índole es mala, no vale ningún esfuerzo. Otro ejemplo claro de ello aparece en el texto español Deberes, de José Dalmau Carles, que se cita acá para dar testimonio de la impotencia de todos los esfuerzos de regeneración y la perplejidad que le causa al autor la causa de este mal. En la lectura titulada "Quien mal anda...", un padre ejemplar, Don Julián, vive dedicado a la educación de sus hijos. Sin embargo, tiene un hijo llamado Augusto, que tiene instintos rebeldes. No obedecía ni a sus padres ni a sus maestros; era ladrón. Ante las características del padre y los fracasos de los esfuerzos por educar al niño, el autor exclama: "Solo Dios podría, pues, decirnos la causa del fenómeno que en aquel lugar se desarrollaba" (DALMAU, 1906, p. 
134). Ya en la adolescencia, lo encierran en la correccional. Sale, pero reincide, y su padre le dice: "Sal, vete; yo te echo de esta casa honrada. iHas perdido a tus padres para siempre! iEl presidio te espera! iiYo te maldigo!!” (DALMAU, 1906, p. 136). La hermana y la madre interceden y el padre lo acepta nuevamente en casa. Sin embargo, el padre muere al poco tiempo de pena moral mientras que Augusto "continuó rodando por la pendiente del crimen". Finalmente fue condenado a trabajos forzados.

Como se ve, la causa de la maldad de Augusto es un misterio: no es ambiental, tampoco heredada de sus padres, pero sí innata e irremediable. Sin embargo, este caso de un buen padre con un hijo malvado, aunque se encuentra en varias ocasiones, no es frecuente, pues varios autores afirman explícitamente que la causa de estas características, buenas o malas, es la familia:

\begin{abstract}
Es una verdad incontrovertible que las buenas personas hacen las buenas familias, y que, por lógica, donde están las buenas familias se forman las poblaciones buenas de los países educados y civiles.

Allá donde no hubiese más que familias desarregladas, con padres negligentes o viciosos, madres sin amor y de malas costumbres, hijos malcriados y de sentimientos perversos, ¿qué nos tocaría hacer? Escapar enseguida corriendo con nuestro equipaje (PAZZI, 1921, p. 165).
\end{abstract}

En contraste con este determinismo, en varios manuales se menciona el poder de la educación como corrección de los malos deseos e inclinaciones y se encuentran casos de personajes que cambian para bien por la influencia de los padres, los profesores y los buenos compañeros. Por ejemplo, en la célebre serie de libros colombianos de lectura La alegría de leer, en la lectura llamada "La escuela", los autores hacen el elogio de la escuela moderna que permite matar el germen de la ignorancia y evitar que los hombres se hagan juguete de las pasiones y de los tiranos (DE QUINTANA Y QUINTANA, 1930, p. 17). Se reconoce alguna posibilidad de que los niños buenos puedan ejercer una influencia beneficiosa sobre los malos. Sin embargo, en general, en los manuales 
se recomienda a los niños aplicados que no se junten con los malos porque se teme que la influencia de los segundos puede ser mucho mayor que la de los primeros.

Desde inicios de los años treinta, y a medida que avanza el periodo, tanto en Colombia como en España, se reducen las muestras de este sustancialismo (aunque no desaparecen y hay un evidente regreso a ellos durante el primer franquismo) y aumenta la confianza en el poder de la educación. Así mismo, se matizan los ejemplos con los cuales se clasifica a los niños. Por ejemplo, aparecen niños traviesos que, aunque cometen pequeñas transgresiones, en el fondo son 'buenos', o incluso, aunque hayan cometido una falta grave, se arrepienten y logran superar el momento de debilidad. En otros casos, como sucede en algunos manuales franquistas de la postguerra española, el juicio es substancialista y condenatorio, pero no se presentan unas consecuencias tan dramáticas como la cárcel o la muerte del padre; en otros casos, aunque se emite un juicio condenatorio la historia explica los sentimientos del niño malvado y hay cierta simpatía por él. Así sucede en la lectura titulada "El niño de Nazaret" del libro español Segundo libro de lectura de Seix Barral, de 1950, en la que juegan Jesús y Judas niños. Jesús hace unos bellos pájaros de barro que pinta con la luz del sol. Judas no logra imitar el milagro. Jesús se ofrece para pintar los pájaros de Judas, pero éste, lleno de amargura, aplasta sus pájaros con el pie y luego pisa un par de los de Jesús. Es entonces cuando muestra su fondo: "Al levantar Judas el pie y ver el pajarito convertido en una masa de barro, sintió un placer tan perverso, que se echó a reír y levantó el pie para aplastar otro" (SEGUNDO LIBRO DE LECTURA, 1950, p. 47). Jesús entonces hace volar al resto de sus pajaritos. Entonces Judas se echa a llorar a los pies porque, de todos modos, amaba a Jesús. La historia termina con María consolando a Judas arrepentido.

En términos generales, el sustancialismo es más frecuente en los manuales en los que predomina una visión religiosa en la que se tiende a asociar con la bajeza de sentimientos a los deseos y placeres relacionados implícita o 
explícitamente con el sexo. La metáfora predominante en ellas para denotar la relación ilícita con el placer sexual es la de la mancha. A la inversa, el ideal de la infancia como paraíso en el que no hay presencia alguna de sexo se expresa en el concepto de pureza, del cual es arquetipo la virgen María, y secundariamente las santas, las madres, el espacio familiar y los mismos niños. A estos últimos se los debe mantener, por tanto, en un completo aislamiento respecto del tema, de lo cual es ejemplo el texto español citado abajo:

Niños a quienes la curiosidad conduce en alas de la imaginación a buscar el proceso de las cosas que se cuentan y se observan; cuando vuestra inocencia no comprenda el arcano o el misterio de las cosas de este mundo, seguid en vuestra candorosa ignorancia (AZPEURRUTIA, 1931, p. 27).

Del ideal ascético de la pureza, fruto de una educación cristiana, se siguen los que son considerados como 'auténticos' placeres y la felicidad real: "La educación cristiana es el solo elemento de felicidad que hay en la tierra; es garantía de la dulce paz del alma y del reposo apacible del corazón" (LECTURAS GRADUADAS. LIBRO TERCERO, 1931, p. 223).

La sustancialización de la identidad emocional de los niños es menos frecuente, aunque tampoco está ausente, en los libros de lectura donde se manifiesta una visión naturalizada del sexo, de corte un poco más laico (es infrecuente la ausencia total de referencias a la religión católica o a sus representantes y ritos), producto de la penetración del discurso médico higienista. En tal caso la presencia del sexo se hace presente de forma difusa a propósito de la energía física, principalmente de los varones, para los cuales, se sostiene, el deseo sexual, correctamente administrado, es la fuente del apasionamiento y la tenacidad que encausa las actividades humanas, y particularmente aquellas que hacen crecer a los pueblos, como el trabajo y la milicia. Es el caso de algunos manuales escritos durante la Segunda República en donde, además de que se reduce la tendencia a clasificar de forma fija a los 
niños, es más frecuente que se hable de instintos e impulsos con un vocabulario que pretende ser más científico y cercano a las preocupaciones por la raza y la herencia de los higienistas. En dichos manuales, así como aquellos en los que predomina la misma corriente en Colombia, quienes más fuertemente reciben juicios deterministas fatalistas son los alcohólicos y sus hijos. También por esta vía se encuentra la prevención con el placer sexual, no en general, sino el desordenado, que se encuentra en el ambiente de los ebrios, enmarcado por la vileza del lupanar y la vergüenza de las enfermedades.

Esta última tendencia fue frecuente también en los manuales escolares que produjeron en la corriente pedagógica de la Escuela Nueva, en los que las recomendaciones higienistas fueron recurrentes. En ellos la visión sobre la infancia era más optimista y es notorio cómo se hace menos frecuente la imagen del niño esencialmente malo. Sin embargo, la tendencia determinista no desaparece del todo porque se mantiene en la figura del vicioso empedernido, y también en el perezoso.

Esta tendencia menos sustancialista es la que predomina hacia el final del periodo en ambos países en la medida en que se van integrando los placeres y las comodidades a la imagen cotidiana de la vida urbana de las clases medias, que es a quienes tienden a dirigirse gran parte de los manuales que entonces se escribieron.

\section{CONCLUSIONES}

¿Qué función cumplen estos apelativos de la identidad infantil? En una primera lectura, son indicaciones acerca de los comportamientos lícitos, frente a los censurables, así como de los signos corporales de la emotividad educada para la obediencia. De acuerdo con esto, el personaje "niño de buen o mal corazón” es ejemplar en el sentido pleno de la palabra. Sin embargo, yendo un poco más hondo, para el niño real que puede ser descrito siguiendo estos 
modelos, lejos de ser una descripción de su ser, es la imposición de un carácter de ficción, de una personificación. Tal personificación es útil a los agentes de autoridad para hacer inteligibles a los niños, ya que permite clasificarlos según sus emociones, sus posturas corporales, según el uso que le den a su ropa o a sus útiles escolares, según el lenguaje y el tono que usen, según sus aficiones, sus amistades, sus gustos, etc. Adicionalmente, las categorías son extrapolables de los niños a los padres, de los padres a las familias y de las familias a los barrios, los grupos sociales, las razas, los géneros, los grupos profesionales, etc. Finalmente, estas categorías permiten remontarse hasta los pueblos y las culturas y hacerlas inteligibles como si se tratara de personajes.

Hacer algo inteligible consiste en sintetizar las diferencias singulares en conceptos unificados que funcionan como reglas desde las cuales se interpreta la variedad de los fenómenos. En virtud de esto la inteligibilidad propicia una anticipación y homogenización de la realidad. Los conceptos se forman a partir de la sedimentación de experiencias individuales, sociales y culturales y tienden a endurecerse, a fijarse desde la experiencia compartida por una comunidad en un momento histórico. De este modo, las categorías pasan de ser meros instrumentos para describir los fenómenos a ser las pautas de la comprensión y, consecuentemente, sirven de base a las decisiones y las acciones. El marco de inteligibilidad, por tanto, determina toda la realidad que rodea a los individuos, pero también el 'interior de las personas', pues sus sentimientos 'privados' son pensados según las reglas con las que 'se piensa'; esto es, con las categorías que hacen inteligible el mundo. Adicionalmente, como los conceptos constituyen la forma de los fenómenos, se asume que la naturaleza es como los conceptos la determinan. De hecho, desde un realismo acrítico se llega a pensar que los conceptos son así a causa de que 'así son las cosas' en la naturaleza.

De vuelta al tema en los libros de lectura, en la referencia al 'fondo' del niño de hecho se está creando ese fondo. Se está asumiendo que existe un enmascaramiento en los comportamientos, un resorte profundo y oscuro de las decisiones y acciones. Claramente, un otro que acecha constantemente y se 
manifiesta en los momentos decisivos. Al relacionar al otro con el instinto, ese otro se convierte en 'natural', se encarna en el mismo cuerpo y las fuerzas que lo habitan y determinan de forma irrecusable, como las demás fuerzas de la naturaleza. Se debe tener presente que el punto aquí no es que objetivamente exista el 'otro', sino que estos apelativos lo crean y le invisten de una potencia inagotable e invencible. De hecho, el apelativo mismo es el que guarda la potencia para producir al sujeto y la performatividad, el modo de actuación, que le es correspondiente. Se ve en ello un procedimiento semejante al que describe Judith Butler en relación con la producción de la performatividad característica del género:

Es posible que tengamos una expectativa similar [a la creada en el sujeto por la anticipación de la aplicación de la ley] en lo concerniente al género, de que actúe una esencia interior que pueda ponerse al descubierto, una expectativa que termina produciendo el fenómeno mismo que anticipa. [...] La postura de que el género es performativo intentaba poner de manifiesto que lo que consideramos una esencia interna del género se construye a través de un conjunto sostenido de actos, postulados por medio de la estilización del cuerpo basada en el género. De esta forma se demuestra que lo que hemos tomado como un rasgo «interno» de nosotros mismos es algo que anticipamos y producimos a través de ciertos actos corporales, en un extremo, un efecto alucinatorio de gestos naturalizados (BUTLER, 2007, p. 17).

Del mismo modo, los apelativos de los libros que examinamos encausan la mirada de los agentes de poder que creen confirmar en cada actuación del niño aquello que están buscando. Es una regla de interpretación que, además de crear esa identidad profunda, de una vez le da una forma inteligible, hasta tal punto que permite hacer predicciones sobre ella, como vimos en los ejemplos. El aspecto más inquietante del tema se produce en los niños lectores que eventualmente fueron llamados con estos apelativos, pues en el contexto de la confianza que los niños pudieron haberle dado a la palabra de los adultos y a la palabra escrita de los libros de texto, una expresión como 'tienes un mal fondo' puede resonar hasta el punto en el cual el niño vea encarnado en sí mismo el 
personaje que se ha revelado a sus padres o sus maestros, y que él mismo vea confirmada esa expectativa en sus sentimientos, sus decisiones y actuaciones. En cada instancia de confirmación el individuo pudo haber cristalizado el entrecruzamiento de miradas que marcaban su cuerpo y su índole. Esta repetición de la expectativa y el cumplimiento de la misma es la que lleva a afirmar que un individuo es 'bueno', 'perverso' o 'vicioso', a creer que su 'interior' lo es y que la educación solo atempera esta realidad.

Se ha mencionado anteriormente que la revelación de la identidad emocional del niño y de un 'fondo' instintivo permite hacer una naturalización de la índole cuya fuente son las fuerzas del cuerpo. En ese marco, el instinto sexual es protagonista por atribuírsele un origen exclusivamente corporal y por considerarlo como un fenómeno difuso y ubicuo, que, precisamente por ello, se sospecha como motivación transversal que condiciona todas las conductas humanas, en lo que tienen de animales. Como señala Michel Foucault, fue precisamente la ubicuidad y equivocidad del sexo, junto con la atribución a él de un poder causal inagotable y polimorfo lo que le dio tanta potencia a su control y tanto interés como clave de interpretación del individuo (FOUCAULT, 1976, p. 82-85). En la creencia en la fuerza de aquella verdad determinante de la conducta es donde precisamente se halla la proliferación de mecanismos de operación del dispositivo de sexualidad.

Así, la declaración de la existencia del fondo de la identidad infantil ofreció una motivación para investigarlo en el comportamiento y el cuerpo de los niños y, consecuentemente, para convertir a los maestros, los sacerdotes, los médicos, los padres y a los mismos niños en especialistas en su indagación y detección. Asimismo, la escuela en todas sus dimensiones, como espacio físico y simbólico, se convirtió en el escenario adecuado para revelar las identidades infantiles, lo que justificó el asedio del que fueron víctimas los niños en relación con su corporalidad y su intimidad. El resultado de esas pesquisas es la segregación y jerarquización de los niños de acuerdo con sus identidades con la respectiva distribución de privilegios, que en los textos se muestran con la tácita 
o explícita preferencia por parte de padres y maestros, la asignación de premios, las buenas calificaciones o las conversaciones afectuosas y los gestos de aprecio. Estos privilegios, a su vez, sirvieron de estímulo para la competencia de los niños por hacer real la ejemplaridad de los personajes de las lecturas.

Se ve, entonces, como la cultura escolar está atravesada por el mecanismo de las identidades frente al cual su actuación consistió en provocar la realidad de la identidad emocional por el hecho mismo de buscarla. La corrección de los signos censurables estuvo, por tanto, fundada en el temor a delatar una naturaleza ruin y, consecuentemente, la escuela sirvió como el escenario del escarnio y la vergüenza de los niños desobedientes. Oscilando entre uno y otro modelo estaban la mayoría de los niños, cuyo talento principal ha debido ser, en medio de esta atmósfera, la obediencia sin reparos a la autoridad y el control y el disimulo de los deseos y los placeres.

Como se ha mostrado, hubo una disminución significativa del uso de las atribuciones de una identidad fija para los niños en los libros de lectura colombianos de los años cuarenta y cincuenta, y en los libros de lectura españoles de los años cincuenta, esto es, posteriores al periodo inicial de la dictadura franquista. Sobre los motivos de este cambio de actitud solo tenemos algunas hipótesis que podrán ser examinadas en investigaciones posteriores. El cambio puede deberse a varias causas simultáneas: en primer lugar, la influencia y mayor penetración de los discursos de pedagogos de la Escuela Nueva que tenían una concepción dinámica de la infancia, y del ser humano en general, en virtud de la cual se le otorgó un peso mucho mayor al ambiente para la formación de los niños que a la índole individual (HERRERA, 1999; OSSENBACH; ZULUAGA, 2004). Adicionalmente, varios de estos reformadores educativos fueron maestros cuya práctica pedagógica en el aula de clase, junto con la intención de acercarse a la ciencia y de darle mayor peso a la experiencia misma con los niños, los hizo más sensibles a las diferencias no solo entre los diferentes niños, sino a los cambios de cada niño a lo largo de su historia escolar. En segundo lugar, en el periodo se produce un tránsito, en los dos 
países, desde sociedades predominantemente rurales a sociedades urbanas. Las emergentes clases medias urbanas, público mayoritario de los manuales escolares, empiezan a distanciarse de la ahora percibida como rígida moral ascética del pasado, en la cual estas etiquetas, los 'salvos' y los 'condenados', eran tan frecuentes. La pequeña burguesía urbana perece haber sido más consciente de los matices de los deseos y placeres y más optimista con respecto a los efectos de la educación para lograr los efectos deseados por el poder en los individuos y las poblaciones. En tercer lugar, es patente en los mismos libros de lectura un cambio de comprensión de la naturaleza de la infancia. Desde una visión en la que el niño es representado como un adulto pequeño se pasa a una representación del niño como un ser esencialmente diferente del adulto que vive en su propio mundo, un lugar que es idealizado como un paraíso depurado de las inquietudes de los adultos. La liberación de las etiquetas nos parece consecuente con este cambio en la comprensión de la infancia, pues estas identidades, particularmente las pasiones y los instintos, estaban claramente asociadas con las realidades del mundo adulto.

Queda la duda para una investigación posterior de si estas atribuciones de identidades emocionales fijas verdaderamente desaparecieron progresivamente de la cultura escolar, junto con las connotaciones de escarnio y vergüenza que ellas implicaron, o si se han metamorfoseado en otras más sutiles, pero igualmente operativas.

\section{REFERENCIAS}

ARIAS, Francisco. Alma española. Barcelona: Ruiz Romero, 1939.

AZPEURRUTIA, José Maria. Escucha niño. Libro de lectura variada integrado por composiciones ofrendadas a los niños españoles por varios de los mejores escritores que tiene hoy España e ilustrado por varios de los más afamados dibujantes y pintores. Madrid: Tipografía Yagües, 1931.

BACCA, Ángela; RAMÍREZ, Julián. Representaciones y prácticas en el 
campo de las relaciones de pareja en Bogotá en el siglo XX: tránsitos entre la tradición y la modernidad. 2003. Tesis (Maestría en Sociología) Departamento de Sociologia, Universidad Nacional de Colombia, Bogotá, 2003.

BERNAL, Rodolfo. Libro de lecturas escogidas en prosa y en verso para niños y niñas. 19. ed. 1942. Bogotá: Voluntad, 1891.

BIDEGAÍN, Ana. Control sexual y catolicismo. In: VELÁSQUEZ-TORO, Magdala (dir.). Las mujeres en la historia de Colombia. Volumen 3. Bogotá: Norma, 1995, p. 120-146.

BOLINAGA, Josefina. Mi costurero. Libro de lectura para niñas. 3. ed. Plasencia: Sánchez Rodrigo, 1963.

BUTLER, Judith. El género en disputa. El feminismo y la subversión de la identidad. Barcelona: Paidós, 2007.

CONDE-CALDERÓN, Jorge (ed.). Nación, educación, universidad y manuales escolares en Colombia: tendencias historiográficas contemporáneas. In: Coloquio colombiano de historia de la educación, 4, 2001, Barranquilla. Anais... Barranquilla: Fondo de Publicaciones de la Universidad del Atlántico, 2001.

CORTÁZAR, Roberto; RENGIFO, Francisco M.; HERRERA, Antonio Otero. Nuevo lector colombiano. 32. ed. 1965. Bogotá: Voluntad, 1913.

DALMAU, José. Deberes. Barcelona: Dalmau Carles, Pla S. A., 1906.

DALMAU, José. El primer manuscrito. Método completo de lectura. No numerada. 1949. Barcelona: Dalmau Carles, Pla S. A., 1925.

DE QUINTANA, Susana; QUINTANA, Evangelista. La alegría de leer. Libro cuarto. 6. ed. 1938. Bogotá: Voluntad, 1930.

ESCOLANO, Agustín (ed.). Historia ilustrada del libro escolar en

España. 2 vols. Madrid: Fundación Germán Sánchez Ruipérez, 1997.

FOUCAULT, Michel. Historia de la sexualidad. 1. La voluntad de saber. México: Siglo XXI, 1976.

GÓMEZ TUTOR, Raimundo. Pepe tercero. 17. ed. 1930. Madrid: Librería y Casa Editorial Hernando, 190?.

GUEREÑA, Jean-Louis; OSSENBACH, Gabriela; DEL POZO, María del Mar. Manuales escolares en España, Portugal y América Latina (siglos XIX y XX). Madrid: Uned, 2005. 
GUEREÑA, Jean-Louis (ed.). La sexualidad en la España contemporánea 1808-1950. Cádiz: Universidad de Cádiz, 2011.

HERNÁNDEZ DÍAZ, José María. Espacios escolares, contenidos, manuales y métodos de enseñanza. In: GUEREÑA, Jean-Louis; TIANA-FERRER, Alejandro; RUIZ-BERRÍO, Julio. Historia de la educación en la España contemporánea. Diez años de investigación. Madrid: Ministerio de Educación y Ciencia. C.I.D.E., 1994, p. 191-213.

HERRERA, Martha. Modernización y Escuela Nueva en Colombia: 19141951. Bogotá: Universidad Pedagógica Nacional, Plaza y Janés Editores Colombia, 1999.

LECTURAS GRADUADAS. Libro tercero. Barcelona: F.T.D, 1931.

MARIN, Álvaro; CANO, Alejandro. Mi cuarto libro de lectura. Medellín: Félix de Bedout e Hijos, 1952.

NOGUERA, Carlos. Discurso médico y prácticas higiénicas durante la primera mitad del siglo XX en Colombia. Medellín: Eafit, 2003.

OSSENBACH, Gabriela; SOMOZA-RODRIGUEZ, Miguel. Los manuales escolares como fuente para la historia de la educación en América Latina. Madrid: Uned, 2001.

OSSENBACH, Gabriela; ZULUAGA, Olga Lucía. Modernización de los sitemas educativos Iberoamericanos. Siglo XX. Tomo II. Bogotá: Magisterio, 2004.

PASCUAL DE SAN JUAN, Pilar. Escenas de familia (continuación de Flora). Libro de lectura en prosa y en verso para niños y niñas. Barcelona: Imprenta Elzeveriana y Librería Camí, 1891.

PAZZI, Juan. El amigo. 11. ed. 1932. Barcelona: Juan Ruiz Romero, 1921.

PEDRAZA, Zandra. En cuerpo y alma: visiones del progreso y la felicidad. Bogotá: Universidad de los Andes, 1999.

PÉREZ, Jesús. El discurso pedagógico relativo a la sexualidad en España (1940-1962). Madrid: Editorial de la Universidad Complutense de Madrid, 1993.

PÉREZ, Jesús. Sexualidad y hegemonía social. La pugna por el ordenamiento sexual en España durante la primera mitad del siglo XX. Revista de sexología, n. 73, 1996, número monográfico. 
PINILLA, Alexis; SAUZA, Luz Marina; HERRERA, Martha. La identidad nacional en los textos escolares de ciencias sociales. Colombia 19001950. Bogotá: Universidad Pedagógica Nacional, 2003.

RESTREPO MEJÍA, Martín. La escuela colombiana. Libro II. Edición de 1923. Bogotá: Imprenta de La Luz, 1918.

RINCÓN-VERDUGO, Cecilia. La enseñanza de la lectura y la escritura en Colombia, 1870-1936. Una mirada desde la práctica pedagógica. Bogotá: Universidad Distrital Francisco José de Caldas, 2003.

ROA, Paola. Salud, cuerpo y vida. Una genealogía de la educación sexual en la escuela colombiana. Praxis y saber, p. 67-84, 2017.

SÁENZ, Javier; SALDARRIAGA, Óscar; OSPINA, Armando. Mirar la infancia: pedagogía, moral y modernidad en Colombia, 1903-1946. Medellín: Editorial Universidad de Antioquia; COLCIENCIAS; Uniandes; Foro Nacional por Colombia, 1997.

SEGUNDO LIBRO DE LECTURA. 2. ed. 1951. Barcelona: Seix-Barral, 1950.

SEOANE, José. El placer y la norma: genalogía de la educación sexual en la España contemporánea: orígenes (1820-1920). Barcelona: Octaedro, 2006.

SERRANO-LÓPEZ, Federico Guillermo. Estrategias para acceder a los puntos ciegos del dispositivo de sexualidad en los libros de lectura para niños en España y Colombia entre 1900 y 1960. Espacio, Tiempo y Educación, 2, n. 1, 2015b, p. 141-162. DOI: http://dx.doi.org/10.14516/ete.2015.002.001.008.

SERRANO-LÓPEZ, Federico Guillermo. Formar madres y padres. La preparación para la sexualidad en los manuales escolares en España y Colombia entre 1930 y 1960. Tesis (doctorado en Pensamiento Español y Latinoamericano) - Departamento de Antropología Social y Pensamiento Filosófico Español, Universidad Autónoma de Madrid, Madrid, 2013.

SERRANO-LÓPEZ, Federico Guillermo. Madres e hijos enamorados. La sublimación de la relación maternal en los libros de lectura españoles y colombianos de la primera mitad del siglo XX. Historia y Memoria de la Educación, n. 2, 2015 a , p. 219-237. DOI: 10.2594.45/6h6m8e.0.29.9270.1551.3154013.

SERRANO-LÓPEZ, Federico Guillermo; SOMOZA-RODRÍGUEZ, Miguel. Social Constructs regarding the physical and sexual energy of whites, indigenous South Americans and blacks in Spanish and Colombian primary school reading books 
between 1900 and 1960. History of Education, 46, n. 5, 2017, p. 578-594. DOI: 10.1080/004676oX.2017.1337934.

TIANA-FERRER, Alejandro. Espacios escolares, contenidos, manuales y métodos de enseñanza. Madrid: Uned, 2000.

TIANA-FERRER, Alejandro. La investigación histórica sobre los manuales escolares en España: el Proyecto Manes. Revista Clío y Asociados, la historia enseñada, n. 4, p. 101-119, 1999.

URREGO, Miguel. Sexualidad, matrimonio y familia en Bogotá 18801930. Bogotá: Planeta, 1997.

VALENTÍN O EL NIÑO BIEN EDUCADO. 5. ed. 1938. Madrid: Ediciones Bruño, 19??.

VÁSQUEZ, Francisco; MORENO, Andrés. Genealogía de la educación sexual en España. De la pedagogía ilustrada a la crisis del estado de bienestar. Revista de Educación, n. 309, p. 67-94, 1996.

VÁSQUEZ, Francisco; MORENO, Andrés. Sexo y razón. Una genealogía de la moral sexual en España (siglos XVI-XX). Madrid: Akal, 1997.

VILLALAÍN, José Luis. Manuales escolares en España. Madrid: Uned, 1997.

FEDERICO GUILLERMO SERRANO LÓPEZ es profesor e investigador del Departamento de Humanidades y Filosofía de la Universidad del Norte. Es doctor en pensamiento español y latinoamericano de la Universidad Autónoma de Madrid.

E-mail: federicol@uninorte.edu.co

(i) http://orcid.org/0000-0002-0720-8571

Recebido em: 27 de novembro de 2017

Aprovado em: 13 de outubro de 2018

Revista História da Educação - RHE

Associação Sul-Rio-Grandense de Pesquisadores em História da Educação - Asphe

Artigo de acesso aberto distribuído nos termos de licença Creative Commons. 